\title{
Hypergolic ignition modulated by head-on collision, intermixing and convective cooling of binary droplets with varying sizes
}

\author{
Dawei Zhang ${ }^{\mathrm{a}, \mathrm{b}, 1}$, Dehai Yu ${ }^{\mathrm{a}, 1}$, Peng Zhang ${ }^{\mathrm{a}, *}$, Yueming Yuan ${ }^{\mathrm{b}}$, Lianjie Yue ${ }^{\mathrm{b}}$, Taichang Zhang ${ }^{\mathrm{b}}$, \\ Xuejun Fan ${ }^{\mathrm{B}}$ \\ a Department of Mechanical Engineering, The Hong Kong Polytechnic University, Hung Hom, Kowloon, Hong Kong \\ ' State Key Laboratory of High Temperature Gas Dynamics, Chinese Academy of Sciences, Beijing, People's Republic of China
}

\section{A R T I C L E I N F O}

\section{Article history:}

Received 2 March 2019

Received in revised form 9 May 2019

Accepted 15 May 2019

Available online 20 May 2019

\begin{abstract}
A B S T R A C T
The hypergolic ignition induced by the head-on collision of TMEDA and WFNA droplets was experimentally investigated with emphasis on the effect of droplet size on the ignitibility and the ignition delay time. The ignitibility regime nomogram in $W e-d_{0}$ space indicates that the reduction of droplet size tends to suppress the hypergolic ignition. The ignition delay time, which was precisely determined by using grayscale level analysis, becomes shorter for smaller droplets. The seemingly conflicting size effects were resolved by means of time scaling analysis to reveal the size dependence of the three pre-ignition processes, which were identified as the first stage of droplet collision, deformation and intermixing, the second stage of droplet heating from interior to surface, and the third stage of droplet vaporization subject to heat loss by convective cooling.
\end{abstract}

(c) 2019 Elsevier Ltd. All rights reserved.

\section{Introduction}

Hypergolic propellants are widely adopted in rocket engines to simplify the engine design, to allow multiple restarts, and thereby to improve the maneuverability. In rocket engine combustor, the impinging jets of the initially separated fuel and oxidizer generate numerous colliding droplets, which subsequently undergo a series of physicochemical processes involving heat and mass transfer in both liquid- and gas-phase and eventually cause gas-phase ignition without requiring any external ignition sources [1-3]. The ignition delay time (IDT) of hypergolic propellants is often defined by the lapse of time between the initial contact of the droplets and the emergence of visible luminous kernel (often accompanied by rapid heat release) in gas phase.

Being of less toxicity and comparable specific impulse and ignition delay, $\mathrm{N}, \mathrm{N}, \mathrm{N}^{\prime}, \mathrm{N}^{\prime}$ - tetramethylethylenediamine (referred to as TMEDA hereinafter) has been tested as an alternative hypergolic fuel $[4,5]$, with white fuming nitric acid (referred to as WFNA hereinafter) as oxidizer, to replace the acutely toxic and potentially carcinogenic hydrazine and its derivatives. Many experimental methods have been developed to investigate hypergolic ignition. Being different in the extent of liquid-phase mixing, the piston-driven rapid mixing apparatus was designed to minimize

\footnotetext{
* Corresponding author.

E-mail address: pengzhang.zhang@polyu.edu.hk (P. Zhang).

1 The first and second authors contributed equally to this paper.
}

the mixing effect in liquid phase [6,7], the impinging jet apparatus often employs partial mixing $[8,9]$, and the drop test uses a free or suspended propellant drop to impact the liquid pool of another propellant either confined in a container or placed on a surface $[10,11]$. Despite that important understandings have been obtained through these experimental methods, they have a common deficiency that it is generally difficult to quantify the system-dependent liquid-phase mixing owing to the unavoidable "wall effect" by the jet nozzle or the container or the surface of the devices. Experimental method free from any "wall effect" is evidently desirable for resembling droplet-collision-initiated hypergolic ignition in real systems.

The authors have successfully established a droplet collision apparatus [12] for hypergolic propellants and conducted an experimental study on binary, head-on collision of free droplets of TMEDA and WFNA, with emphasis on the effects of Weber number and size ratio on hypergolic ignitability and IDT. It was found that the hypergolic ignition is critically dependent on the exothermal liquid-phase reactions of TMEDA and WFNA, mainly through the proton transfer reaction, TMEDA $+2 \mathrm{HNO}_{3} \rightarrow$ TMEDADN [13-16], whose rate is controlled by the effective liquid-phase mixing of droplets by collision. With the help of time-resolved shadowgraph imaging, three distinct pre-ignition stages were observed and will be briefly summarized as follows.

Stage I is characterized by droplet collision, coalescence and deformation. Because of the relatively low droplet temperature and short time, chemical reactions are minimal in this stage and 
droplet behaviors are controlled by fluid dynamical processes such as droplet coalescence, deformation, and oscillation. The Weber number, $W e=\rho_{O} d_{O} U^{2} / \sigma_{0}$, and the size ratio, $\Delta=d_{O} / d_{F}$, are the controlling non-dimensional parameters [17-19], where $d$ the diameter of droplet, $\rho$ the liquid density, $\sigma$ the surface tension coefficient, $U$ the droplet impact velocity; the subscripts " $F$ " and "O" denote TMEDA and WFNA, respectively.

Stage II is characterized by droplet heating and slow vaporization (compared with Stage III). Droplet heating is inferred by observing the blurring of droplet surface and the expansion of opaque vapors. Because the exothermic liquid-phase reactions occur in the interior of the merged droplet where two propellants intermingle and mix, the droplet temperature is being increased by heat transfer from the interior to the surface, rendering that vaporization is relatively slow in the stage.

Stage III is characterized by rapid vaporization and reactions. A large amount of vapors and gaseous species are accumulated around the droplet and to be ignited at the end of this stage. The faster vaporization is evident in the shadowgraph images as a quickly expanded large opaque area. The following ignition and combustion in gas phase last for a few milliseconds and leave behind nonflammable condensed-phase products.

In order to focus on quantifying the effects of droplet collision and mixing dynamics [18-20] on the hypergolic ignitability and IDT and to avoid dealing with many factors at one time, the previous work of the authors deliberately limits its scope to studying the effects of $W e$ and $\Delta$ by fixing $d_{0}=1.45 \mathrm{~mm}$ [12]. The ignitability regime nomogram in the parameter space of $W e-\Delta$ shows that the hypergolic ignition favors large $W e$ (for enhanced droplet mixing which facilitates the subsequent heating and vaporization processes) and small $\Delta$ (for more adequate liquid-phase reactions which required appropriate fuel/oxidizer volume ratio). For the same reason, the IDT has an overall tendency of decreasing with increasing We. The effects of $\Delta$ on IDT can be understood from two aspects: increasing of $\Delta$ facilitates the internal mixing [21] and hence favors the ignition; increasing of $\Delta$ deviates the binary system cubically from the chemical stoichiometry of the liquidphase reaction thus suppresses the ignition.

In spite of the above worthy results, which reveal the controlling effects of collision dynamics and intermixing on the hypergolic ignition of TMEDA/WFNA, the effects of droplet size (other than those of the size ratio) were not considered, and therefore the validity of the above results under more general and practically useful situations merits the present study. In the previous study [12], interesting preliminary results have been reported that no ignition was observed for smaller WFNA droplets $\left(d_{0}=0.3 \mathrm{~mm}\right)$ while other parameters retained unchanged. We hypothesized that if the droplet sizes are too small, the liquid-phase reactions may not generate sufficient amount of heat to vaporize the liquids. In addition, the large surface-volume ratio of smaller droplets increases the heat loss to the environment, again slowing down the liquid vaporization.

\section{Experimental setup and measurement methodology}

The experimental apparatus is schematized in Fig. 1 and has been described in great detail in our previous paper [12]. Therefore, only brief explanation is given below. The bipropellant WFNA and TMEDA droplets were generated by two sets of independently controlled nozzles, which were connected to well-sealed oxidizer and fuel liquid tanks and driven by ultrapure Nitrogen gas regulated by pressure regulator. The solubility of nitrogen gas in both TMEDA and WFNA is negligible under our experimental conditions, and therefore the pressure can be precisely regulated. Due to the strong corrosiveness of WFNA, an in-house needle-shape nozzle made of anti-corrosion material of Teflon (Polytetrafluoroethylene) was used to generate the WFNA droplet to move downwardly.

Compared to the WFNA droplet generator used in our previous study [12], the size of the WFNA droplet could be precisely controlled and varied in the present study. The droplet size herein is primarily dependent on the diameter of the nozzle office and secondarily on the supply pressure of the nitrogen gas. The size of the generated WFNA droplet decreases with increasing pressure of pressurized gas and also with the generating frequency up to $30 \mathrm{~Hz}$. For a generated WFNA droplet, the interference from its preceding and following droplets can be avoided by precisely adjusting the collision frequency by means of precisely controlling the generating frequency of TMEDA droplets. TMEDA droplets were dispensed horizontally from a custom-made micro electromagnetic valve. The droplet size is mainly controlled by the diameter of orifice of the valve and moderately affected by the switch onand-off time duration and the liquid flow rate. Diameters of generated WFNA and TMEDA droplets have an uncertainty less than $6 \%$. The binary collision of droplets can be accurately realized and

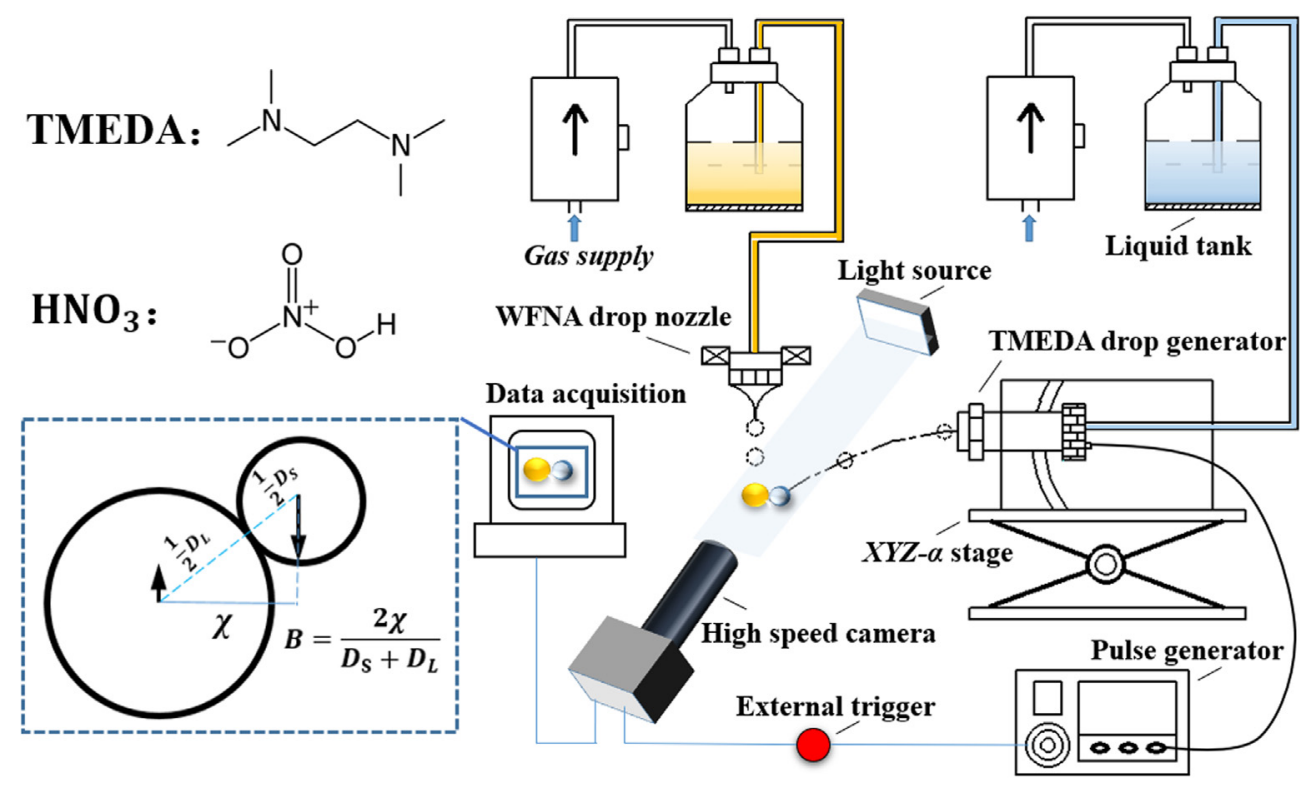

Fig. 1. Schematic of the experimental apparatus for hypergolic ignition by binary collision of TMEDA and WFNA droplets. 
controlled by adjusting the dispensing trajectory of TMEDA droplet through a micrometric XYZ stage with spatial resolution of $2 \mu \mathrm{m}$ and also by continuously regulating the dispensing frequency and the signal delay.

In the present study, the size of WFNA droplets can vary from $0.9 \mathrm{~mm}$ to $1.5 \mathrm{~mm}$ instead of being fixed at $1.45 \mathrm{~mm}$ in the previous study. Such a variation of WFNA droplet size was found to be sufficiently large to produce distinct phenomena to be presented and analyzed in the following sections, while it is sufficiently small to rule out the possible influence of Ohnesorge number, $O h=\mu_{0} / \sqrt{\rho_{0} \sigma_{0} d_{0}}$, a dimensionless measure of droplet viscosity $(\mu)$. It is recognized that the fluid-dynamic influence of viscosity on the droplet coalescence and mixing and in turn the hypergolic ignition is not negligible [12]. However, Oh is substantially small as being of $O\left(10^{-3}\right)$ in the present problem and varies too slightly (by less than 30\%) to cause any significant difference.

High speed camera, Phantom V711, was employed to record the temporally resolved shadowgraph images with imaging speed of 5000 frames per second and exposure time of $5 \mu \mathrm{s}$. The diameters and relative velocity of WFNA and TMEDA droplets are determined from the shadowgraph images by using the MATLAB software. The uncertainties of droplet diameter are less than $7 \%$ and those of velocity are about 3\% 8\%. As shown in Fig. 1, the uncertainty of droplet trajectories from head-on collision is measured by the impact parameter, $B=x / L<0.1$, where $x$ is the projection of the separation distance $L$ between the droplet centers in the normal direction to the relative velocity.

\section{Phenomenological description of hypergolic ignition}

Figure 2 shows the time-resolved shadowgraph images of the hypergolic ignition induced by binary collision of droplets for $d_{0}=1.3 \mathrm{~mm}, 1.1 \mathrm{~mm}$ and $0.9 \mathrm{~mm}$. In the three cases, $W e=61$ and $\Delta=1.5$ are fixed so that comparison can be made with the representative case described in detail in the previous study [12], where Weand $\Delta$ are the same but $d_{0}=1.45 \mathrm{~mm}$. It is noted that different reference lengths were employed in the images for a clear presentation of the entire processes, which spatially expand from $O\left(10^{-1}\right) \mathrm{mm}$ to $O(10) \mathrm{mm}$. In spite of the size variation, the three pre-ignition stages summarized in the Introduction can still be observed:

In Stage I, the two droplets collide and deform with clear droplet surfaces that are similar to those exhibited in collision of nonreactive droplets [19]. Moreover, the time duration is almost the same for Stage I, which ends at near $5.0 \mathrm{~ms}$ for all three cases. This implies that the dynamics of droplet collision and mixing in Stage I follows the fluid dynamic similarity, and droplet sizes enter the problem through the non-dimensional parameters such as We and $\Delta$.

In Stage II, as the merged droplet being heated up by the exothermic liquid-phase reactions, opaque vapor is emitted outwardly from the droplet surface, which is gradually concealed, as shown from $6.0 \mathrm{~ms}$ to $18.0 \mathrm{~ms}$ in Fig. 2(a). An interesting observation is that, for the case of $d_{0}=0.9 \mathrm{~mm}$ shown in Fig. 2(c), the droplet surface has already been mostly blurred at $9.0 \mathrm{~ms}$, which is earlier than the $12 \mathrm{~ms}$ for the case of $d_{0}=1.1 \mathrm{~mm}$ in Fig. 2(b) and earlier than the $18 \mathrm{~ms}$ for the case of $d_{O}=1.3 \mathrm{~mm}$ in Fig. 2(a). This indicates that decreasing the size results in shorter Stage II, probably because the smaller droplet is more readily heated up.

In Stage III, the droplet vaporization becomes significantly faster for all the three cases. Compared with those in Stage II, the shadowgraph images in Stage III must be zoomed out by about three times to show the entire process. The size effect on the duration of the stage is not evident but the eventual ignition, character-

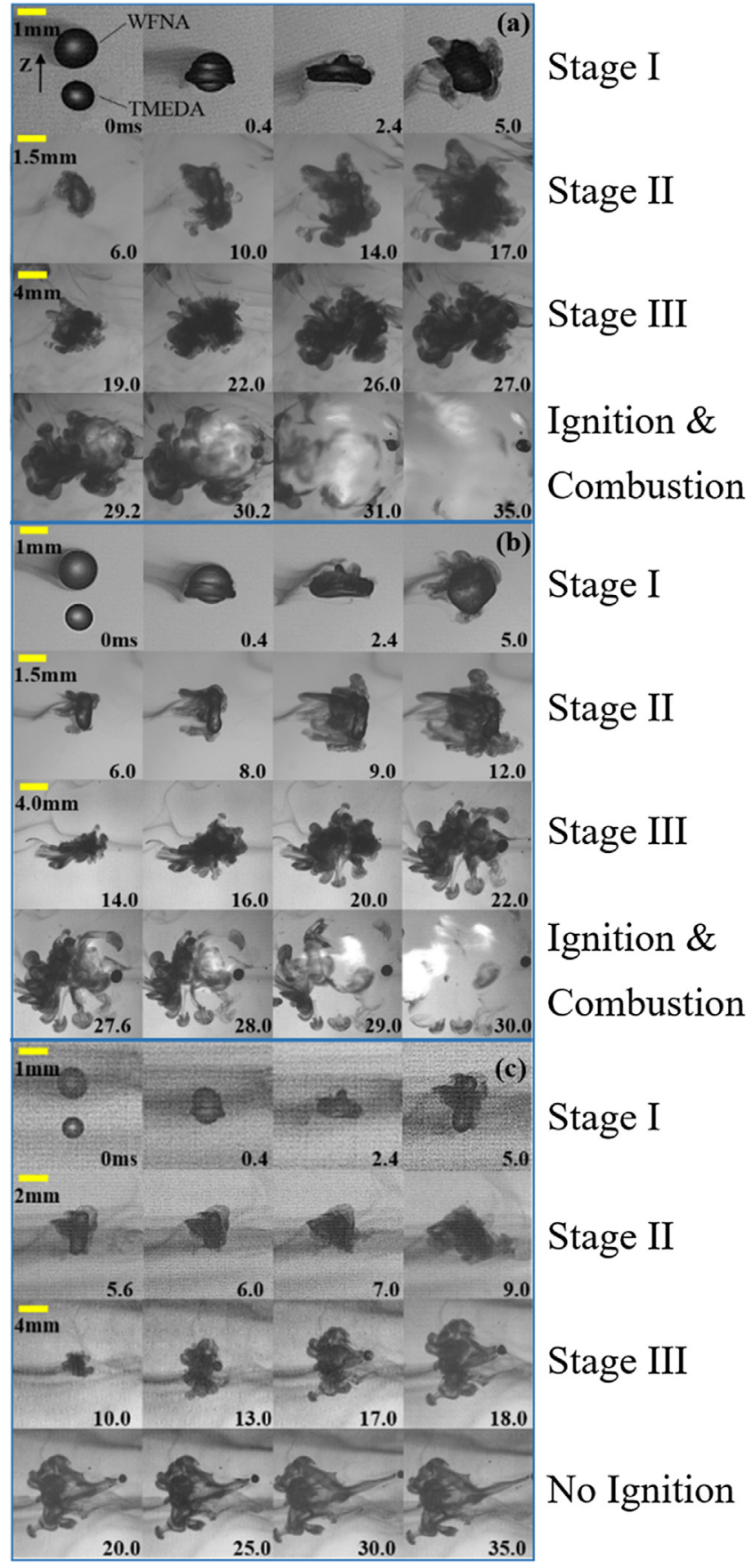

Fig. 2. Shadowgraph images of the hypergolic ignition by droplet collision at $W e=61$ and $\Delta=1.5$. WFNA droplet diameter $d_{O}$ is (a) $1.3 \mathrm{~mm}$, (b) $1.1 \mathrm{~mm}$, and (c) $0.9 \mathrm{~mm}$.

ized by the emergence of luminous flame kernel, occurs at $27.6 \mathrm{~ms}$ for the case of $d_{0}=1.1 \mathrm{~mm}$, being earlier than that for the case of $d_{0}=1.3 \mathrm{~mm}$. In addition, the ignition does not occur for the case of $d_{0}=0.9 \mathrm{~mm}$, showing that the small reduction of the droplet size by $30 \%$ is enough to bring the system from ignitability to nonignitability.

\section{Limiting phenomena of hypergolic ignition with various droplet sizes}

Comprehensive parametric study was conducted over wide ranges of We from 20 to 100 and of $d_{O}$ from $0.9 \mathrm{~mm}$ to $1.5 \mathrm{~mm}$, 


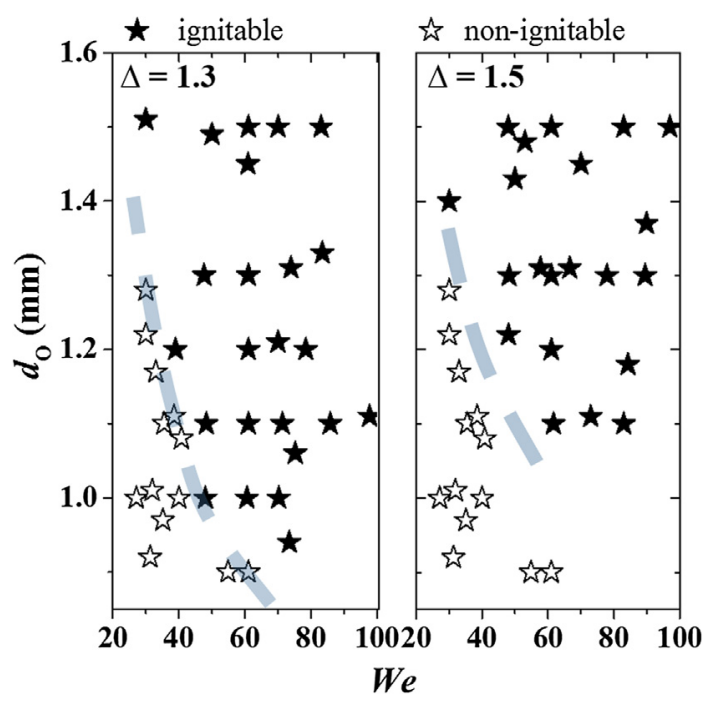

Fig. 3. Ignitability regime nomogram in $W e-d_{0}$ parameter space for $\Delta=1.3$ and $\Delta=1.5$.

for $\Delta$ being 1.3 and 1.5. A regime nomogram for hypergolic ignitability in $W e-d_{O}$ space is shown in Fig. 3.

For $\Delta=1.3$, a fitted curve divides the $W e-d_{0}$ parameter space into two regimes with the ignitable regime being above the curve and the non-ignitable regime below. According to the nomogram, it is evident that the ignition favors higher We as the size $d_{0}$ decreases, implying that reducing the droplet size tends to suppress the ignition and therefore a larger $W e$ is required to promote the intermixing and reactions between the fuel and oxidizer droplets. For $\Delta=1.5$, the same observations can be made to the size effect on the ignitability. At the larger size ratio, the nonignitable regime is slightly extended to higher $W e$, which is consistent with the previous results [12].

\section{Size effect on hypergolic ignition delay}

\subsection{Precise determination of IDT by grayscale analysis}

To accurately determine the ignition delay time, a method based on the analysis of grayscale levels on the shadowgraph images, which has already been employed and verified in our previous study [12], is adopted and briefly summarized as follows:

The grayscale in a shadowgraph image can be divided into 256 levels denoted by $G$, in which $G=0$ corresponds to the pixels representing completely opaque area and $G=255$ to the completely bright. Following the same criterion adopted in our previous study [12], the grayscale of the image background is defined by $G=100$, the luminous ignition kernel by $G>G_{\text {high }}=250$, and the opaque vapors by $G<G_{\text {low }}=5$. The total number of pixels on a shadowgraph image is counted as $N$, that of the luminous kernel as $N_{b}$, and that of the opaque vapors as $N_{d}$. Therefore, the ignition occurrence is identified by an abrupt rise of $N_{b} / N$ with time, as shown in Fig. 4. As an example, the IDT for the case (a) in Fig. 2 can be unambiguously determined as $29.0 \mathrm{~ms}$, when $N_{b} / N$ starts to rapidly rise while $N_{d} / N$ starts to suddenly drop. The grayscale analysis method has been verified to be insensitive to the threshold values differentiating the dark and bright regions from the background. Specifically, for different pairs of threshold values, $\left(G_{\text {high }}, G_{\text {low }}\right)$, such as $(245,10)$ and $(235,20)$, the uncertainty in quantifying the IDT is less than $0.2 \mathrm{~ms}[12]$.

It should be noted that, in case (a) Fig. 2, the droplet is completely covered by the dark vapors when vaporization takes place,

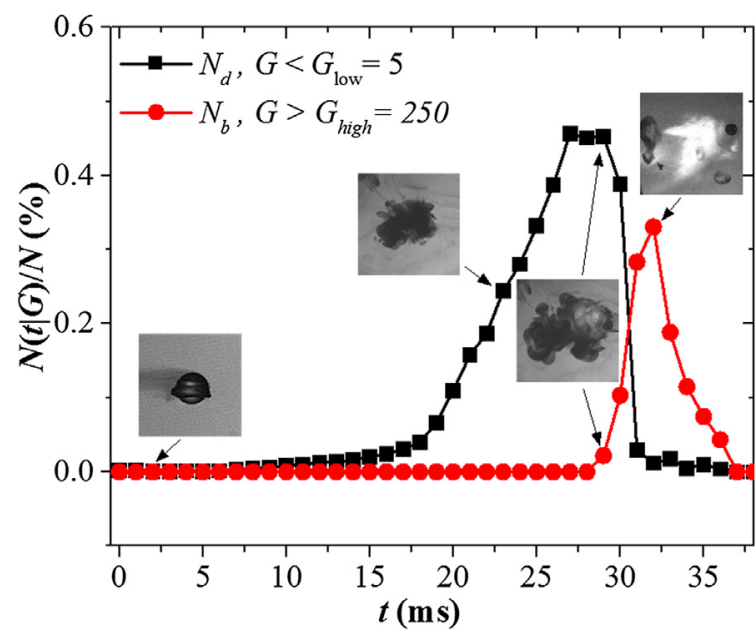

Fig. 4. Precise determination of IDT by grayscale level analysis for the case (a) in Fig. 2.

such as $17 \mathrm{~ms}$, which prevents direct observation to the droplet. The abrupt increase of dark area in our gray-scale analysis actually refers to the rapid accumulation of the gas-phase reactants, which should not be misunderstood as the droplet size increase, or the pressure and temperature rise as appears in other ignition events such as those in shock tubes.

\subsection{Decrease of IDT with droplet size reduction}

Fig. 5 shows the variation of IDT with $W e$ at $\Delta=1.3$ and 1.5 and for various $d_{0}$. Consistent with the previous findings [12], the IDT generally tends to decrease with increasing $W e$, because of the mixing enhancement of TMEDA and WFNA within the merged droplet.

As shown in Fig. 5, at a first glance, the shrinking of the droplet size tends to reduce the IDT as indicated by the falling of the curves characterizing dependence of IDT upon We as decreasing $d_{0}$. Nevertheless, it should be recalled that the ignition cannot be achieved for the case of $\Delta=1.5$ with $d_{0}=1.0 \mathrm{~mm}$, verifying that the reduction of droplet size tends to suppress the ignition as indicated in the regime diagram of Fig. 4. It is recognized that the above seemingly conflicting size effects on the ignition delay time

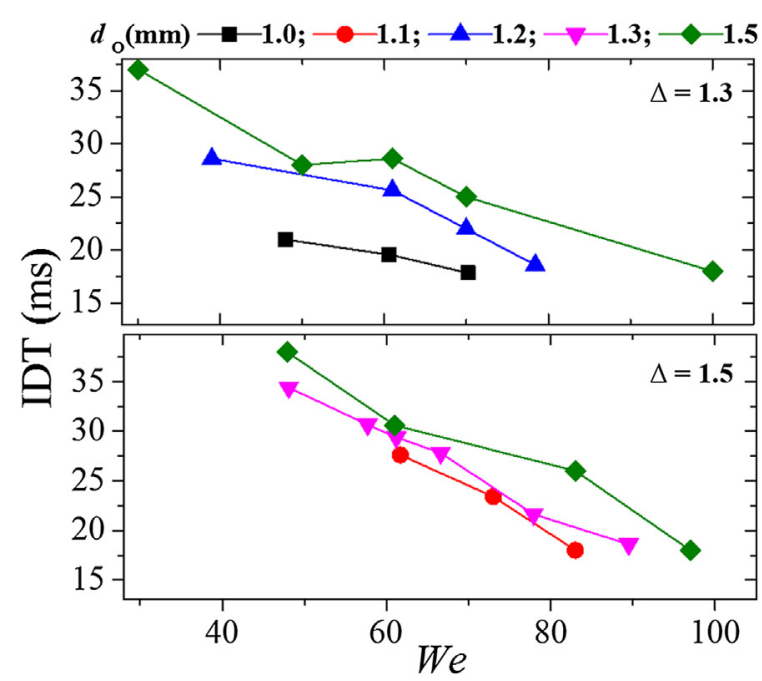

Fig. 5. Variation of IDT (in ms) with $W e$ at $\Delta=1.3$ and $\Delta=1.5$ and for various $d_{O}$ of $1.0 \mathrm{~mm}, 1.1 \mathrm{~mm}, 1.2 \mathrm{~mm}, 1.3 \mathrm{~mm}$ and $1.5 \mathrm{~mm}$. 
and the ignitability are caused by using dimensional times (in millisecond), which may distort the correct physical pictures of flow similarity. The various pre-ignition processes occur on different time scales, as have been described in Section 3, and consequently their dependence on the droplet size are controlled by different physicochemical laws. In the following section, we shall conduct a time scale analysis to the pre-ignition processes to resolve the size effects.

\section{Time scaling analysis of intermixing, heating and vaporization stages.}

\subsection{Characteristic time scales of intermixing, heating and vaporization} stages

As discussed in Section 3, Stage I is dominated by the fluid dynamic processes of droplet collision, deformation and intermixing. As shown in Fig. 2, there is little gas-phase reactant evaporated from the droplet surface, so we can infer that the thermal and chemical processes, such as heat generation due to liquid phase chemical reaction and heat conduction within the droplet, are negligible in the collision stage. The characteristic time scale for Stage I, denoted by $\tau_{I}$ or $\tau_{\text {impact }}$, can be formally written as a function of the controlling flow similarity parameters, such as We and $\Delta$ in the present problem,

$\tau_{I}=\tau_{\text {impact }}=f(W e, \Delta)$

It is seen that $\tau_{I}$ is the same for a given set of these nondimensional parameters, through which $d_{0}$ exerts its influence.

In stage II, the internal motion within tends to slow down because the droplet collision and the followed droplet oscillations usually last for a couple of milliseconds [21]. The chemical heat release due to liquid-phase reactions and the heat conduction from the droplet interior to the droplet surface become significant. This can be inferred from Fig. 2 that there is a considerable amount of evaporated gas-phase reactant during stage II. We assume that the liquid-phase chemical reaction is much faster than the heat conduction so that the latter process plays the controlling role. The relevant physical properties for scaling analysis should be the liquid thermal conductivity, $\lambda_{l}$, the liquid density $\rho_{l}$, the constant-volume heat capacity $c_{v, l}$, and the diameter of the droplet $d_{0}$, in terms of which we can write a time scale in the form of

$\tau_{I I}=\tau_{\text {heating }} \sim \frac{d_{O}^{2}}{\lambda_{l} / \rho_{l} c_{v, l}}$

which can be interpreted as the time scale of heating, i.e. the time lapse from the heat transport from the inside to the surface of the merged droplet.

For hypergolic fuels, the characteristic time scale for chemical reaction tends to be much more rapid than those interpreting the transport processes, such as mass diffusion and heat conduction. Hence, the ignition of hypergolic fuel takes place spontaneously when they were brought into contact by various transport processes. Therefore, the time duration for the heating stage is determined by the heat conduction properties of the droplet, i.e. the heat release from liquid-phase reaction does not appear in the expression of $\tau_{\text {heating }}$ in Eq. (2).

As the heating has raised the droplet temperature to sufficiently high levels, a significant vaporization takes places over the surface of the droplet in stage III, which is responsible for the majority of the accumulation of the fuel and oxidizer vapor to be mixed and ignited. Stage III is characterized by a considerable regression of the droplet surface, which implies that droplet cooling must be considered as a result of the convective heat loss to the cold ambience as increasing the surface-volume ratio.
A precise calculation of the vaporization time-scale $\tau_{\text {III }}$ requires solving the complete energy equation of the droplet with convective heat loss being properly considered, but this is beyond the scope of the present study. Alternatively, we adopt an approximate approach. First, we considered an ideal situation in which the cooling effect was completely absent, so the time scale for the steady vaporization of the droplet can be obtained from the well known $d^{2}$-Law,

$d_{0}^{2} / \tau_{\text {vaporization }}\left(\lambda_{g} / \rho_{l} c_{p, g}\right) \ln \left(1+B_{h, v}\right)$

where $\lambda_{g}$ is the thermal conductivity and $c_{p, g}$ the constant-pressure heat capacity of the gas-phase products. In the present experiment, the vaporization on the droplet surface is sustained by the heat release resulting from the liquid-phase reaction inside the droplet, which creates a temperature difference between the interior and the surface of the droplet. Consequently, $B_{v}=c_{p}\left(T_{\text {internal }}-T_{s}\right) / q_{v}$ is the variant of heat transfer number, where $q_{v}$ is the latent heat of vaporization; $T_{\text {internal }}$ is the internal temperature of the droplet, which is affected by the heat release of liquid-phase reaction; $T_{s}$ is the surface temperature of the droplet.

Note that if we change the present TMEDA/WFNA system to other propellant pair and/or the surrounding temperature, $B_{h, v}$ can be moderately changed. However, the logarithmic function $\ln \left(1+B_{h, v}\right)$ of $B_{h, v}$ tends to considerably alleviate that change and does not significantly influence the scaling relation between the vaporization time and the droplet diameter.

Recognizing that the heat loss increases with the surfacevolume ratio, we estimated the time scale characterizing the cooling process, denoted by $\tau_{\text {cooling }}$, in the form of [22]

$\tau_{\text {cooling }} \sim \frac{c_{p, g} \rho_{g}}{(S / V) h}=\frac{c_{p, g} \rho_{g}}{6 \lambda_{g}} \frac{1}{N u} d_{O}^{2}$

where $\rho_{g}$ is the density of the gas phase, $S / V \sim 6 / d_{O}$ the surfacevolume ratio of the droplet, and $h$ the phenomenological convection heat transfer coefficient, which is related to the Nusselt number through $h=N u \lambda_{\mathrm{g}} / d_{0}$. By approximating the droplet as a sphere, the Nusselt number is related to the Reynolds number, $R e=\rho_{g} U_{l} d_{O} / \mu_{g}$, by $N u \sim R e^{1 / 2}$ [22], where $U_{l}$ refers to the velocity of the merged droplet in the gas phase, $\mu_{g}$ the dynamic viscosity of the gas phase.

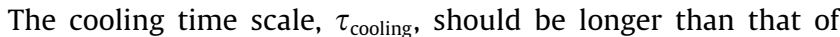
vaporization, $\tau_{\text {vaporization }}$, otherwise the vaporization process would be substantially suppressed or even terminated due to sufficiently strong cooling of the droplet. Furthermore, the time scale of vaporization with cooling, $\tau_{\text {III }}$, must be larger than that of the vaporization without cooling, $\tau_{\text {vaporization }}$, because the presence of cooling tends to elongate the actual time scale of the vaporization process. Therefore, we hypothetically interpret the actual vaporization time scale as a proper average of $\tau_{\text {cooling }}$ and $\tau_{\text {vaporization }}$ in above considered limiting cases. We adopted the weighted power mean, which can be regarded as the generalized geometric mean, to formally specify the actual time scale as

$\tau_{\text {vaporization-cooling }}=\tau_{\text {vaporization }}^{1-p} \tau_{\text {cooling }}^{p}$

where $p$ is regarded as a fitting parameter, with magnitude in the range $[0,1]$, and its precise value can be determined by fitting the experimental results. Evidently, the actual vaporization time scale specified by Eq. (5) satisfies the physical consideration that $\tau_{\text {vaporization-cooling }}>\tau_{\text {vaporization }}$. It is noted that the weighted-power mean may not be the only choice for the scaling relation. In fact, linear relations $\tau_{I I I}=c_{1} \tau_{\text {vaporization }}+c_{2} \tau_{\text {cooling, }}$, where $c_{1}$ and $c_{2}$ are constants, may be also viable. The exact functional relation cannot be determined in the framework of scaling analysis, while the 
weighted-power-mean gives us considerable mathematical convenience in the following analysis.

Substituting Eq. (3) and Eq. (4) into Eq. (5), we obtained

$\tau_{\text {vaporization-cooling }} \sim \frac{c_{p, g} \rho_{l}^{1-p} \rho_{g}^{p}}{6^{p} \lambda_{g}} \frac{1}{N u^{p}} d_{O}^{2}$

Recalling the Frossling relation of the Nusselt number, which is approximately written as

$N u=2+0.5 R e^{1 / 2}$

since the cubit root of Prandtl number can be assumed as unity with sufficient accuracy for the present scaling analysis. Substituting Eq. (7) in Eq. (6), we have

$\frac{1}{N u^{p}}=\frac{1}{\left(2+0.5 R e^{1 / 2}\right)^{p}}=\frac{1}{0.5^{p}(R e)^{p / 2}}\left(1+\frac{4}{R e^{1 / 2}}\right)^{-p}$

Because $p<1$ and $\operatorname{Re} O(100)$ the bracket term on the right-hand side of (8) is close to unity and we arrived at

$\tau_{\text {vaporization-cooling }} \sim \frac{c_{p, g} \rho_{l}^{1-p} \rho_{g}^{p}}{6^{p} \lambda_{g}} \frac{1}{0.5^{p}(R e)^{p / 2}} d_{O}^{2}$

Substituting the definition of Reynolds number $R e=\rho_{g} u_{g} d_{O} / \mu_{g}$ into Eq. (9), we finally have

$\tau_{\text {vaporization-cooling }} \sim \frac{c_{p, g} \rho_{l}{ }^{1-p} \rho_{g}^{p / 2} \mu_{g}^{p / 2}}{3^{p} \lambda_{g} u_{g}^{p / 2}} d_{O}^{2-p / 2}$

In terms of which, we can evaluate the surface regression rate $K_{r}$ of the droplet, characterizing the vaporization process,

$K_{r} \sim \frac{d_{0}^{2}}{\tau_{\text {vaporization-cooling }}} \sim \frac{3^{p} \lambda_{g} u_{g}^{p / 2}}{c_{p, g} \rho_{l}^{1-p} \rho_{g}^{p / 2} \mu_{g}^{p / 2}} d_{O}^{p / 2}$

\subsection{Size effects during intermixing, heating and vaporization stages.}

As discussed in Section 4, the number of pixels with $G<G_{\text {low }}$, $N_{d}$, can be approximately regarded as the occupied area of the vapor. Consequently, the volume occupied by the vapor can be estimated by $\alpha_{N} N_{d}^{3 / 2}$, where $\alpha_{N}$ characterizes the geometry effect, and $\left(N_{d} / N\right)^{3 / 2}$ can be used to approximately measure the total volume of the accumulated vapor around the merged droplets as the result of vaporization.

To elucidate the size effects on the time scales of the preignition processes and thus on the IDTs, we reanalyzed the three representative cases (presented in Fig. 2) at $W e=61$ and $\Delta=1.5$ for different $d_{O}$ by using the results from the above time scaling analysis. As seen in Fig. 6(a), if the physical time scale is adopted, the time lapses of Stage I for different droplet sizes are almost the same, as have been discussed in Section 3. The size effect in Stage I is through the flow similarity parameters, such as We and $\Delta$, which are fixed for the three cases. The obvious deviations of the three cases in Stages II and III can be understood by that the physical time scales change with the droplet sizes.

In Stage II, $\left(N_{d} / N\right)^{3 / 2}$ has a slight increase for all the three cases, as shown in Fig. 6 (a), because the heat generated by the liquidphase reaction is being transferred to the surface, where the temperature is not sufficiently high for vaporization. It is seen that the time lapse of Stage II is shorter for the smaller droplet, which can be hypothetically contributed to the shorter characteristic time for heating, as indicated $\tau_{I I} d_{O}^{2}$ in Eq. (2). To verify this hypothesis, we replotted Stage II in Fig. 6 (b) by using scaled time, $t / d_{0}^{2}$, according to Eq. (2). As we anticipated, all the curves corresponding to differ-
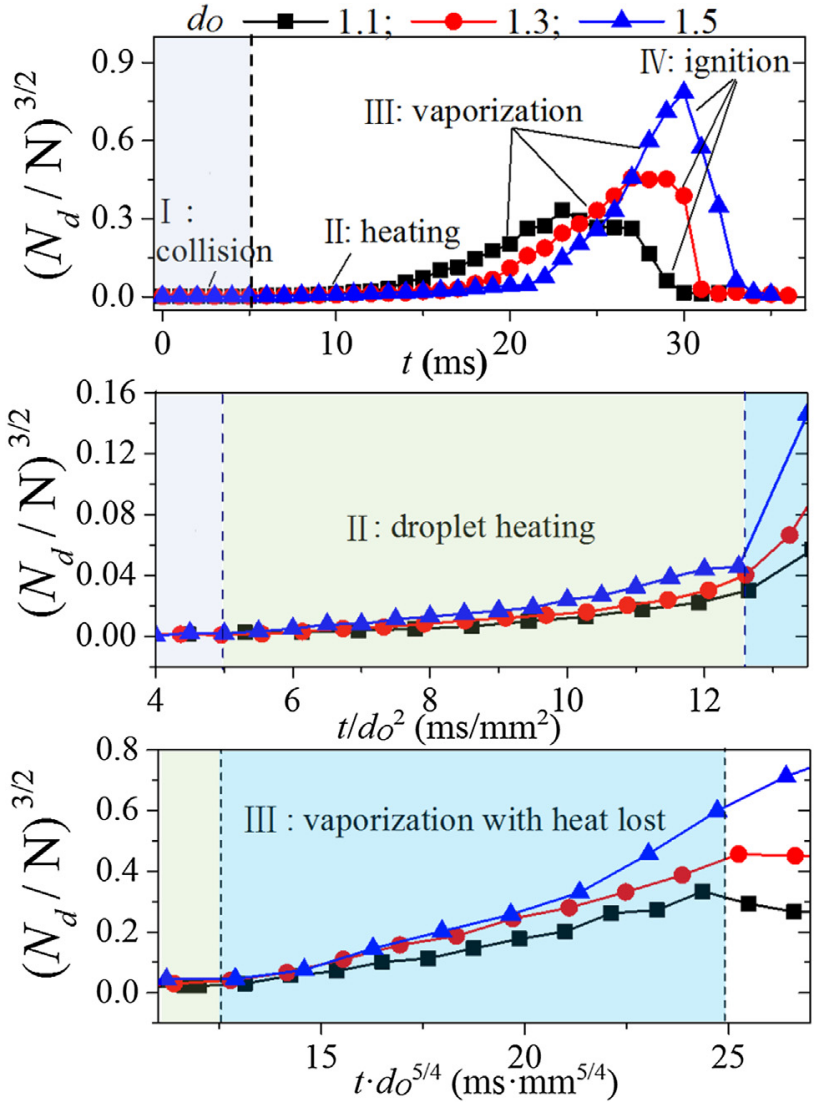

Fig. 6. Size effects on the time scales of the pre-ignition processes at $W e=61$ and $\Delta=1.5$ for various $d_{O}$ of $1.1 \mathrm{~mm}, 1.3 \mathrm{~mm}$ and $1.5 \mathrm{~mm}$.

ent droplet sizes almost collapse into one curve, substantiating the validity of the time scaling estimation.

In Stage III, $\left(N_{d} / N\right)^{3 / 2}$ has a sudden increase due to rapid vaporization, which is characterized by a large slope of the curves in the physical time, as shown in Fig. 6(a). The accumulation rate of the gaseous reactant can be evaluated in terms of the surface regression rate, i.e.

$\frac{d m_{g}}{d t} \sim K_{r} d_{O}$

substituting Eq. (12) into which, one obtains the dependence of $d m_{g} / d t$ on the droplet size, i.e.

$\frac{d m_{g}}{d t} \sim \frac{3^{p} \lambda_{g} u_{g}^{p / 2}}{c_{p, g} \rho_{l}^{1-p} \rho_{g}^{p / 2} \mu_{g}^{p / 2}} d_{o}^{1+p / 2}$

Nevertheless, it should be noted that $\tau_{I I I}$ characterizes the total time lapse for the complete vaporization of the droplet into gasphase, while the ignition in gas phase actually depends upon the mass of the evaporated gas phase reactant. Thus, according to Eq. (13), we can formally derive the time elapse by accumulating unit mass of gaseous phase reactant as

$\frac{d t}{d m_{g}} \sim \frac{c_{p, g} \rho_{l}^{1-p} \rho_{g}^{p / 2} \mu_{g}^{p / 2}}{3^{p} \lambda_{g} u_{g}^{p / 2}} d_{O}^{-1-p / 2}$

Therefore, the time scale characterizing the accumulation of unit mass of gaseous phase reactant should be specified as

$\tau_{\text {accumulation }} \sim m_{g}^{c} \frac{c_{p, g} \rho_{l}^{1-p} \rho_{g}^{p / 2} \mu_{g}^{p / 2}}{3^{p} \lambda_{g} u_{g}^{p / 2}} d_{O}^{-1-p / 2}$ 
where $m_{g}^{c}$ should be regarded as some characteristic unit mass (per gram, or milligram, etc.) of the concerned gaseous phase reactant. According to Eq. (15), the time scale for accumulating unit mass of gaseous phase reactant becomes larger as decreasing the diameter of the droplet, thus retarding, or even eliminating the ignition in gas-phase.

To close the scaling analysis, we should specify the fitting parameter $p$. As an example, we tested our analysis by choosing $p=1 / 2$ and compared with the experimental data. The results in Fig. 6 indicates that our scaling analysis is able to interpret the experimental observations. It should be noted that our analysis is not restricted to $p=1 / 2$, which happens to estimate the vaporization time scale with cooling $\tau_{\text {vaporization-cooling }}$ in terms of geometric average of $\tau_{\text {vaporization }}$ and $\tau_{\text {cooling. }}$.

Based on the above discussion, we can conclude the size effects on the IDTs by examining and comparing the effects on each preignition process. First, the time duration of Stage I is determined by the non-dimensional collision parameters, We and $\Delta$, through which the droplet sizes affect the entire hypergolic processes. Second, the time duration of Stage II is additionally affected by the physics of droplet heating, which give rise to a size-dependent time scale, $\tau_{I I} d_{O}^{2}$, to the problem. For smaller droplets, the reduced droplet heating time tends to cause a shorter IDT. Third, the time duration of Stage III is controlled by the droplet vaporization accompanied by heat loss due to convective cooling, whose time scale is a weaker function of the droplet size, $\tau_{\text {accumulation }} d_{O}^{-5 / 4}$. For smaller droplets, the decreased vaporization rate results in a prolonged vaporization stage, and hence tends to cause a larger IDT. Consequently, with fixed $W e$ and $\Delta$,decreasing $d_{0}$ would cause a substantially shorter Stage II and moderately longer Stage III, which combined to result in a shorter IDT. However, further decreasing $d_{O}$ to sufficiently small value would significantly increase the heat loss in Stage III, which results in insufficiently accumulated vapor in gas phase, leading to non-ignition.

\section{Concluding remarks}

Droplet size effects on the hypergolic ignition by head-on collision of TMEDA and WFNA droplets was experimentally studied and theoretically analyzed. The hypergolic ignitability of the system is characterized by a regime nomogram in the $W e-d_{0}$ parameter space, indicating that decreasing the droplet size $\left(d_{0}\right)$ seems to suppress the ignition so that a larger We or a smaller $\Delta$ or both together is favored for ignition. However, the experimentally observed ignition delay time at fixed $W e$ and $\Delta$ decreases with the droplet size, seemingly implying an opposite size effect on the ignition.

Time scaling analysis to the three pre-ignition stages reveals that the first stage of droplet collision does not attribute to the observed size effects because it is dominantly affected by We and $\Delta$; the second stage of droplet heating is quadratically shortened by reducing the droplet size, consequently causing a shorter ignition delay; the third stage of droplet vaporization with heat loss is prolonged by reducing the droplet size though a scaling of $d_{0}^{-5 / 4}$ if $d_{O}$ is not too small to cause non-ignition by the substantially increased heat lost.

Applying the present understanding into the real engine systems merits future work, such as to precisely identify the actual distribution of droplet sizes and the range of collision parameters. In real rocket engines, droplet collisions with high Weber numbers (beyond $W e=20-100$ considered in the present study) may take place. These collisions lead to droplet shattering, produce satellite droplets of much smaller sizes, and therefore reduce the Weber numbers of subsequent collisions into the range that the present study concerns.

\section{Declaration of Competing Interest}

The authors declared that they have no conflicts of interest to this work.

\section{Acknowledgement}

The work was supported by the Hong Kong RGC/GRF (PolyU 152217/14E and PolyU 152651/16E), NSFC (91641105), and the Hong Kong Polytechnic University (G-YBXN and G-SB1Q). The authors are grateful for an additional support by a collaborative open fund from the Key Laboratory of High-temperature Gas Dynamics, Chinese Academy of Sciences.

\section{References}

[1] E.A. Fletcher, G. Morrell, Ignition in liquid propellant rocket engines, in: M.G.J. Ducerme, A.H. Lefebvre (Eds.), Progress in Combustion Science and Technology, Pergamon, Oxford, London, New York, Paris, 1960, pp. 183-215.

[2] R.J.M. Eric, A. Hurlbert, Propellant Ignition and Flame Propagation, in: V. Yang, M. Habiballah, J. Hulka, M. Popp Inc (Eds.), Liquid Rocket Thrust Chambers: Aspects of Modeling, Analysis, and Design, American Institute of Aeronautics and Astronautics, Inc., Virginia, 2004.

[3] G.P. Sutton, O. Biblarz, Rocket Propulsion Elements, 8th ed., John Wiley \& Sons, Hoboken, New Jersey, 2010.

[4] A.S. Gohardani, J. Stanojev, A. Demairé, K. Anflo, M. Persson, N. Wingborg, C. Nilsson, Green space propulsion: opportunities and prospects, Prog. Aerosp. Sci. 71 (2014) 128-149.

[5] H. Kang, J. Won, S.W. Baek, S. Kwon, Autoignition and combustion characteristics of sodium borohydride-based non-toxic hypergolic fuel droplet at elevated temperatures, Combust. Flame 181 (2017) 149-156.

[6] M. Kilpatrick, L.L. Baker, A study of fast reactions in fuel-oxidant systems: anhydrous hydrazine with 100 percent nitric acid, Symp. (Int.) Combust. (1955) 196-205.

[7] R.L. Schalla, E.A. Pletcher, The behavior of the system triethylamine-white fuming nitric acid under conditions of rapid mixing, Symp. (Int.) Combust. (1957) 911-917.

[8] D. Erik, C. Kevin, P. Timothée, H. Stephen, Ignition of advanced hypergolic propellants, in: 46th AIAA/ASME/SAE/ASEE Joint Propulsion Conference \& Exhibit, AIAA, Nashville, 2010, p. 6984.

[9] S. Wang, S.T. Thynell, A. Chowdhury, Experimental study on hypergolic interaction between $\mathrm{N}, \mathrm{N}, \mathrm{N}^{\prime}, \mathrm{N}^{\prime}$-tetramethylethylenediamine and nitric acid, Energy Fuels 24 (2010) 5320-5330.

[10] E.M. Dambach, B.A. Rankin, T.L. Pourpoint, S.D. Heister, Temperature estimations in the near-flame field resulting from hypergolic ignition using thin filament pyrometry, Combust. Sci. Technol. 184 (2012) 205-223.

[11] S. Wang, S. Thynell, An experimental study on the hypergolic interaction between monomethylhydrazine and nitric acid, Combust. Flame 159 (2012) 438-447.

[12] D. Zhang, P. Zhang, Y. Yuan, T. Zhang, Hypergolic ignition by head-on collision of $\mathrm{N}, \mathrm{N}, \mathrm{N}^{\prime}, \mathrm{N}^{\prime}$ - tetramethylethylenediamine and white fuming nitric acid droplets, Combust. Flame 173 (2016) 276-287.

[13] M.J. McQuaid, W.H. Stevenson, D.M. Thompson, Computationally based design and screening of hypergolic multiamines, Proceedings for the Army Science Conference, Orlando, Florida, 2004.

[14] M. McQuaid, Notional Hydrazine-Alternative Hypergols: Design Considerations, Computationally-Based Property Determinations, and Acquisition Possibilities, US Army Research Laboratory, 2006.

[15] W.-G. Liu, S. Dasgupta, S.V. Zybin, W.A. Goddard III, First principles study of the ignition mechanism for hypergolic bipropellants: $\mathrm{N}, \mathrm{N}, \mathrm{N}^{\prime}, \mathrm{N}^{\prime}-$ tetramethylethylenediamine (TMEDA) and $\mathrm{N}, \mathrm{N}, \mathrm{N}^{\prime}, \mathrm{N}^{\prime}-$ tetramethylmethylenediamine (TMMDA) with nitric acid, J. Phys. Chem. A 115 (2011) 5221-5229.

[16] P. Zhang, L.D. Zhang, C.K. Law, Density functional theory study of the reactions of 2-azido-N, N-dimethylethanamine with nitric acid and nitrogen dioxide, Combust. Flame 162 (2015) 237-248.

[17] N. Ashgriz, J. Poo, Coalescence and separation in binary collisions of liquid drops, J. Fluid Mech. 221 (1990) 183-204.

[18] J. Qian, C.K. Law, Regimes of coalescence and separation in droplet collision, J. Fluid Mech. 331 (1997) 59-80.

[19] C. Tang, P. Zhang, C.K. Law, Bouncing, coalescence, and separation in head-on collision of unequal-size droplets, Phys. Fluids 24 (2012).

[20] C. Rabe, J. Malet, F. Feuillebois, Experimental investigation of water droplet binary collisions and description of outcomes with a symmetric Weber number, Phys. Fluids 22 (2010).

[21] C. Tang, J. Zhao, P. Zhang, C.K. Law, Z. Huang, Dynamics of internal jets in the merging of two droplets of unequal sizes, J. Fluid Mech. 795 (2016) 671-689.

[22] F.A. Williams, Combustion Theory, Cummings Publ. Co, California, 1985. 